increased $(-1.5$-fold, $\mathrm{P}<0.05)$ in the aortae of $\mathrm{PKC} \alpha-/$-ApoE-/mice after 18 weeks of Western diet feeding when compared to ApoE-/- controls. This difference in lesion size is greatest in the athero-resistant descending aorta when compared to the athero-prone aortic arch, suggesting PKC $\alpha$ plays a protective role in atherosclerosis; particularly in athero-resistant vascular beds.

Aortic sinus lesion size was not significantly different between PKC $\alpha-/-A p o E-/-$ and ApoE-/- mice at any time point. However, a significant increase in intimal calcification $(-2$-fold, $\mathrm{P}<0.05)$ accompanied by a decrease in aortic sinus cholesterol cleft/necrotic core area $(-1.5$-fold, $\mathrm{P}<0.05)$ was observed at the 28 week time-point in $\mathrm{PKC} \alpha-/-\mathrm{ApoE}-/-$ mice versus ApoE-/- controls. Thus, loss of PKC $\alpha$ increases aortic sinus calcification in the absence of any effect on lesion size in ApoE-/- mice. To further characterise the calcifications in PKC $\alpha$-/-ApoE-/- and ApoE-/- mice, detailed morphometric analysis of whole brachiocephalic arteries was performed using microCT. Large calcified structures (ranging from $50-850 \mu \mathrm{m}$ ) containing pores (ranging from $8-35 \mu \mathrm{m}$ ) were localised within lesions in the brachiocephalic artery of PKC $\alpha-/-A p o E-/-$ and ApoE-/- mice. These calcifications were localised solely to lesions at the base of the brachiocephalic artery in ApoE-/mice whereas in PKC $\alpha$-/-ApoE-/- mice, additional calcifications were observed at the bifurcation.

In conclusion, our study has identified that $\mathrm{PKC} \alpha$ may play a protective role in both atherosclerosis and intimal calcification. This suggests that inhibiting PKC $\alpha$ may not be of any therapeutic benefit in atherosclerosis. Activation of PKC $\alpha$ could instead represent a new therapeutic target for atherosclerosis-induced intimal calcification.

Conflict of interest None

\section{BS62 MYOCARDIAL FIBROSIS AND THE DEGREE OF GAP JUNCTION COUPLING DIRECTLY MODIFIES THE UNDERLYING MECHANISM OF FIBRILLATION}

Balvinder Handa*, Xinyang Li, Catherine Mansfield, Richard J Jabbour, David Pitcher, Rasheda A Chowdhury, Nicholas Peters, Fu Siong Ng. Imperial College London

\subsection{6/heartjnl-2019-BCS.222}

Background Mechanisms that maintain ventricular fibrillation (VF) remain debated. There are data supporting both critical areas sustaining rotational drivers (RDs) and the contrary hypothesis of disorganised myocardial activation driving VF. Abnormal electrical coupling between cardiomyocytes through gap junctions (GJ) and ventricular fibrosis are major factors in disease related remodelling, and have been implicated in initiation and maintenance of VF. In this study we hypothesised that the mechanism of VF is directly altered by fibrosis and GJ coupling, and that modulating these factors shifts or changes the predominant mechanism of fibrillation along the spectrum between disorganised activity and organised drivers.

Methods VF optical mapping was performed in fifty-five perfused rat hearts with differing degrees and patterns of chronic ventricular fibrosis [compact $(\mathrm{CF}, \mathrm{n}=11)$, patchy $(\mathrm{PF}, \mathrm{n}=13)$ and diffuse $(\mathrm{DF}, \mathrm{n}=11)$, and acute GJ coupling modulation with rotigaptide (RTG, GJ coupling enhancer, 0-80nM, $\mathrm{n}=10$ ) or carbenoxolone (CBX, GJ uncoupler, $0-50 \mu \mathrm{M}$, $\mathrm{n}=10$ ). Fibrillation dynamics were quantified using phase analysis, RD tracking and our novel method of global fibrillation organisation quantification, frequency dominance index (FDI), which is defined as the power ratio of highest amplitude dominant frequency in the frequency spectrum.

A. Representative histology of left ventricular anterior wall

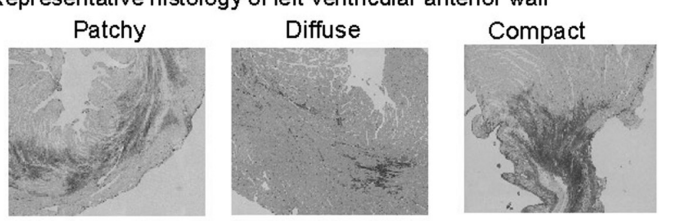

Red $=$ Picrosirius fibrosis stain $/$ Pale yellow $=$ normal myocardium

B. Representative rotational driver (RD) heat map in VF of fibrotic hearts
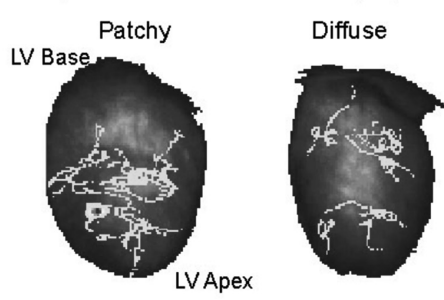

Compact

C. Representative dominant frequency map in VF of fibrotic hearts
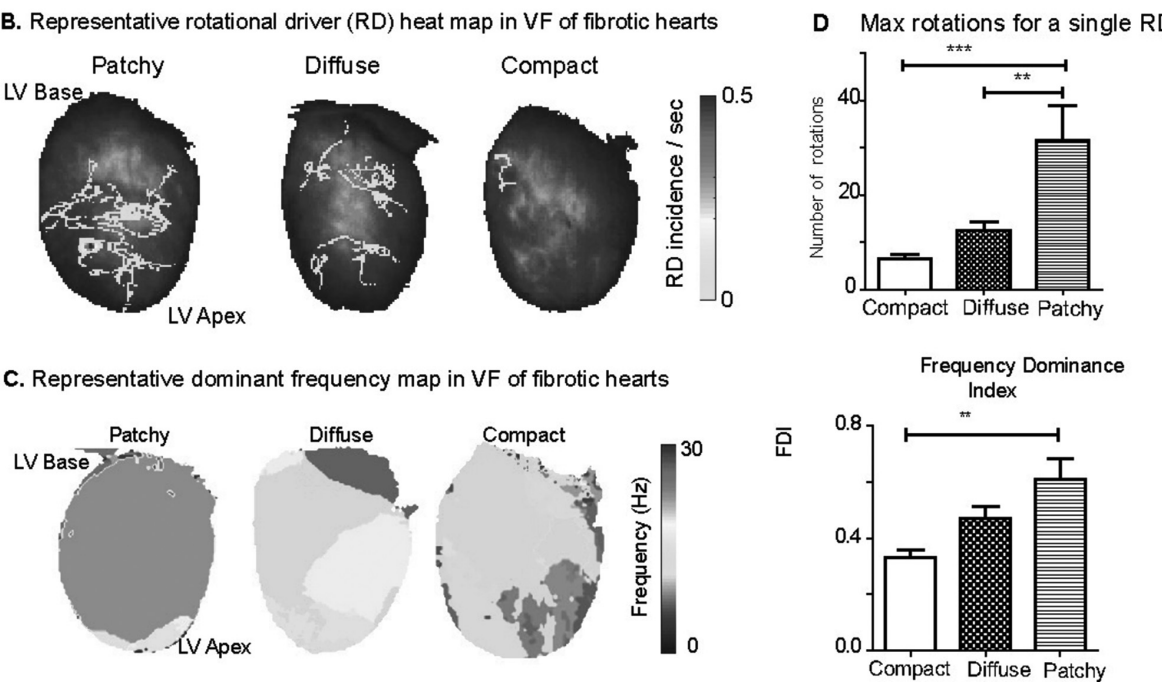

Abstract BS62 Figure 1 
A Representative rotigaptide (RTG) rotation driver (RD) heat map
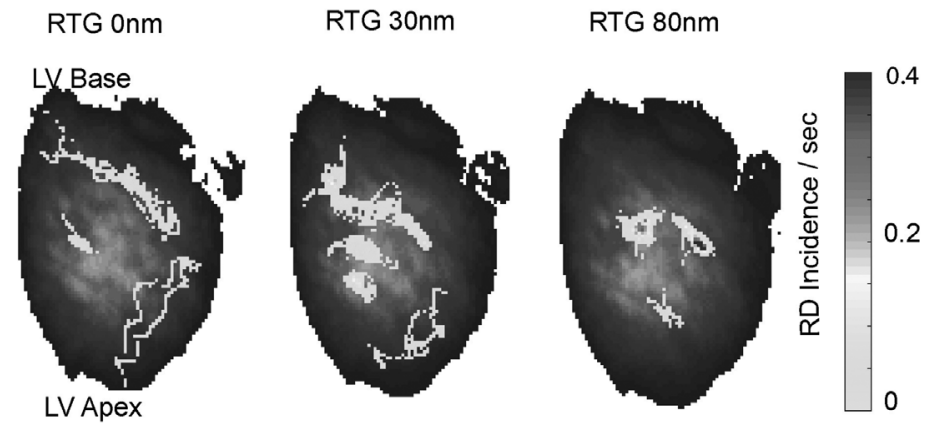

B Representative carbenoxolone (CBX) RD heat map
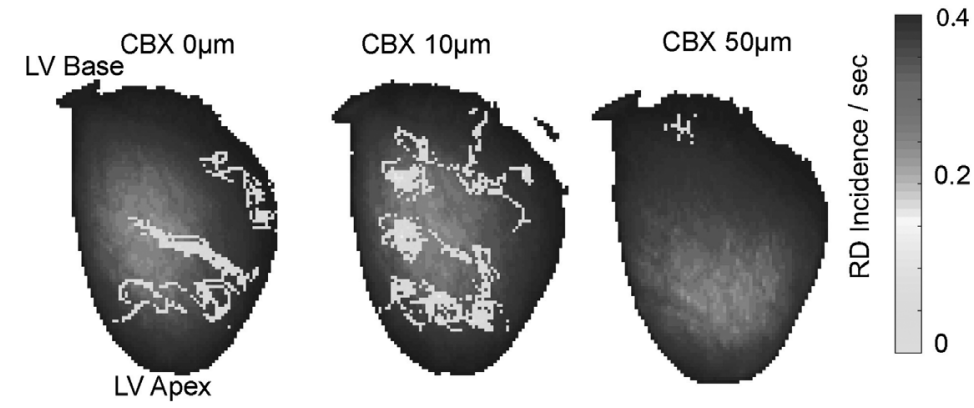

\section{Abstract BS62 Figure 2}

Results In the fibrosis group, VF was driven predominantly by disorganised activity in CF, RDs were detected $26 \pm 7 \%$ of time comparative to $51.2 \pm 4 \%$ in DF and $69.5 \pm 8 \%$ in PF group $(\mathrm{p}=0.001)$. PF stabilised RDs, average maximum rotations for a single RD in $\mathrm{PF}$ were $31.6 \pm 7.1$ comparative to $12.5 \pm 1.7$ in $\mathrm{DF}$ and $6.4 \pm 1.1$ in $\mathrm{CF}, \mathrm{p}<0.001$. VF organisation measured by FDI was higher in PF (PF: $0.61 \pm 0.07$, DF: $0.47 \pm 0.04, \mathrm{CF}$ : $0.33 \pm 0.03, p=0.004$ ) (figure 1). In the GJ modulation group, maximum rotations for a RD increased with RTG $(0 \mathrm{~nm}$ : $5.4 \pm 0.45$ vs $80 \mathrm{nM}: 48.20 \pm 12.32, \mathrm{p}<0.001)$ and decreased with CBX $(0 \mu \mathrm{M}: 8.0 \pm 1.3$ vs $50 \mu \mathrm{M}$ : $0.3 \pm 0.3$, p<0.001). Proportion of time RDs were detected in VF increased with RTG (0nM: $44 \pm 6$ vs $80 \mathrm{nM}$ : 93 $\pm 2, \mathrm{p}<0.001$ ) and decreased with

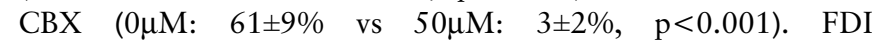
increased with RTG (0nM: $0.53 \pm 0.04$ vs $80 \mathrm{nM}: 0.78 \pm 0.3$, $\mathrm{p}<0.001)$ and decreased with CBX $(0 \mu \mathrm{M}: 0.60 \pm 0.05$ vs $50 \mu \mathrm{M}: 0.17 \pm 0.03, \mathrm{p}<0.001$ ) (figure 2 ).

Conclusion VF mechanisms occur along a spectrum between organised activity with discrete drivers and disorganised myocardial activation. The degree of GJ coupling and ventricular fibrosis are key determinants of the underlying mechanism of VF. Enhanced GJ coupling and patchy fibrosis organised fibrillation and stabilised RDs, whilst GJ uncoupling and compact fibrosis disorganised VF. This study presents a unifying explanation for the numerous mechanisms reported for sustaining fibrillation. Characterising the degree and pattern of fibrosis in patient groups vulnerable to VF might be beneficial in identifying patients with targetable substrate, and GJ modulation might be a potential therapeutic target.

Conflict of interest Nil

\section{BS63 DEVELOPMENT OF E-SENSE: A FLEXIBLE IN VITRO PLATFORM TO DETERMINE CADRIOVASCULAR RISK}

Rhys Smith*. Manchester Metropolitan University

10.1136/heartjnl-2019-BCS.223

R.X. Smith, Alex Langford-Smith, Tristan McKay, Stephen J White

Introduction Coronary heart disease (CHD) is the leading cause of mortality and morbidity globally. Atherosclerosis, a key underlying mechanism in the potentiation of $\mathrm{CHD}$, is often defined as a chronic inflammatory disease provoked by the oxidation of lipids retained in arterial walls resulting in plaque formation. Plaque formations have been demonstrated to preferentially form at bifurcations and arcs exposed to disturbed blood flow patterns (athero-prone), whereas uniform sections exposed to laminar flow (atheroresistant) promote an athero-protective response, implicating the endothelium in the initiation of the disease. Various genes are known to regulate atherosclerosis development; $\mathrm{NF} \kappa \mathrm{B}$ and AP-1 (inflammatory cytokine response), IRF3 (toll-like receptor signalling), XBP1 (unfolded protein response/ER stress) and KLF2 and NRF2 (protective signalling pathways).

Methods Human coronary artery endothelial cells (HCAECs) were immortalised via lentiviral overexpression of anti-senescence polycomb protein, BMI1, which allowed for the extended proliferative lifespan of the primary cells, without affecting their morphology. Immortalised human coronary 